\title{
Is there a connection between sublingual varices and hypertension?
}

\author{
Lennart Hedström ${ }^{1 *}$, Margit Albrektsson ${ }^{1}$ and Håkan Bergh ${ }^{2}$
}

\begin{abstract}
Background: Sublingual varices have earlier been related to ageing, smoking and cardiovascular disease. The aim of this study was to investigate whether sublingual varices are related to presence of hypertension.

Methods: In an observational clinical study among 431 dental patients tongue status and blood pressure were documented. Digital photographs of the lateral borders of the tongue for grading of sublingual varices were taken, and blood pressure was measured. Those patients without previous diagnosis of hypertension and with a noted blood pressure $\geq 140 \mathrm{mmHg}$ and/or $\geq 90 \mathrm{mmHg}$ at the dental clinic performed complementary home blood pressure during one week. Those with an average home blood pressure $\geq 135 \mathrm{mmHg}$ and/or $\geq 85 \mathrm{mmHg}$ were referred to the primary health care centre, where three office blood pressure measurements were taken with one week intervals. Two independent blinded observers studied the photographs of the tongues. Each photograph was graded as none/few (grade 0) or medium/severe (grade 1) presence of sublingual varices. Pearson's Chi-square test, Student's t-test, and multiple regression analysis were applied. Power calculation stipulated a study population of 323 patients.
\end{abstract}

Results: An association between sublingual varices and hypertension was found ( $\mathrm{R}=2.25, p<0.002)$. Mean systolic blood pressure was 123 and $132 \mathrm{mmHg}$ in patients with grade 0 and grade 1 sublingual varices, respectively ( $p<0.0001, \mathrm{Cl} 95 \%$ ). Mean diastolic blood pressure was 80 and $83 \mathrm{mmHg}$ in patients with grade 0 and grade 1 sublingual varices, respectively ( $p<0.005, \mathrm{Cl} 95 \%$ ). Sublingual varices indicate hypertension with a positive predictive value of 0.5 and a negative predictive value of 0.80 .

Conclusions: An association was found between sublingual varices and hypertension. Examining the lateral borders of the tongue is easily done, causes no harm and could be a valuable method for the dental profession to take active part in preventive healthcare.

Keywords: Sublingual varices, Hypertension, Blood pressure, Preventive health care

\section{Background}

Hypertension is the primary risk factor for global disease burden [1] and causes organ damage already in the pre-symptomatic phase, before causing cardiovascular disease such as stroke and myocardial infarction. It is therefore important to discover and treat hypertension as early as possible. In Sweden, there is today no widespread structured screening for hypertension among healthy individuals. However, blood pressure is generally acquired when people are seeking health care for other reasons.

\footnotetext{
* Correspondence: lennart.hedstrom@regionhalland.se

'Public Dental Services, Box 1254, S-43218 Varberg, Sweden

Full list of author information is available at the end of the article
}

A major part of the population visits their dentist on a yearly basis. Approximately $80 \%$ of Swedish grown-ups are in contact with a dental team by a recall system, and about $60 \%$ visit the dental team yearly. Together with a long tradition of prophylactic work, the dental team could potentially be a good screening partner for hypertension by investigating the patient's oral cavity.

A common condition that may be found by the dentist is varicosities, a benign venous lesion. This condition can be found in several locations within the oral cavity; in the buccal mucosa, in the lower lip mucosa, and most often under the lateral borders of the tongue, i.e. sublingual varices [2].

\section{Biomed Central}

(c) 2015 Hedström et al. This is an Open Access article distributed under the terms of the Creative Commons Attribution License (http://creativecommons.org/licenses/by/4.0), which permits unrestricted use, distribution, and reproduction in any medium, provided the original work is properly credited. The Creative Commons Public Domain Dedication waiver (http:// creativecommons.org/publicdomain/zero/1.0/) applies to the data made available in this article, unless otherwise stated. 
Sublingual varices are clinically characterised by small dilated veins under the lateral borders of the tongue. Their pathogenesis may be due to a change in the connective tissue or weakening of the venous wall, as a result of degeneration of elastic fibres related to the ageing process [3-5]. The phenomenon of sublingual varices is not well studied and it may to be correlated with age, smoking and cardiovascular disease and varicose veins of the leg [6-9]. A connection between sublingual varices and hypertension has not yet been shown.

The aim of this study was therefore to investigate whether sublingual varices are related to presence of hypertension.

\section{Method}

\section{Design and setting}

This observational study was performed between May 2010 and February 2013 at the Public Dental Services, Varberg, Sweden.

\section{Study population}

Patients above the age of 40 years were invited to participate in the study in connection to their regular yearly visit. They received both verbal and written information about the study. Those who accepted to participate provided written consent. Exclusion criteria were pregnancy, atrial fibrillation and renal disease [10]. The study was approved by the Regional Research Ethics Committee at the University of Lund (EPN 2009/204), and was performed in accordance with the Helsinki Declaration.

\section{Procedure}

Patients answered a questionnaire about background information (age,sex) and health status (actual smoking, hypertension, atrial fibrillation, ischemic heart disease, myocardial infarction, stroke, other cardiovascular diseases, lower limb varices). One intraoral digital photograph of each lateral borders of the tongue were acquired before the routine oral survey. Thereafter, the patient rested for at least $5 \mathrm{~min}$ in a quiet room. Blood pressure was measured in a sitting position, 2 times in each upper arm using the Korotkoff-Riva-Rocci method, using a cuff, a calibrated manometer and a stethoscope. If a patient had no previous diagnosis of hypertension and the measured average systolic blood pressure was $\geq$ $140 \mathrm{~mm} \mathrm{Hg}$, and/or the average diastolic blood pressure was $\geq 90 \mathrm{~mm} \mathrm{Hg}$, the patient was asked to use a home blood pressure device (Omron M6 Comfort, Omron Healthcare Ltd, Kyoto, Japan) for 1 week [11]. The follow-up blood pressure measurements were acquired in the arm where the highest average blood pressure was noted. If the average blood pressure was equal between arms, the patient decided on his own which arm to apply follow-up measurements. The patient was given both verbal and written instructions on how to use the home blood pressure device [12]. The home blood pressure was measured twice daily, at morning and evening time, with 2 measurements acquired at each time point. The time of acquisition and blood pressure were noted on an enclosed form. The form and home blood pressure monitoring device were returned to the study team for calculating the average blood pressure from day 2-7 [12].

If the average home blood pressure was systolic $\geq 135$ and/or diastolic $\geq 85 \mathrm{~mm} \mathrm{Hg}$ the patient was referred to his ordinary primary health care centre $[10,12]$, where 3 office blood pressure measurements were acquired 1 week apart [13]. If the average office blood pressure was systolic $\geq 140$ and/or diastolic $\geq 90 \mathrm{~mm} \mathrm{Hg}$ the patient was diagnosed with hypertension, and the result was reported back to the study team. An average systolic blood pressure ranging from 140 to $159 \mathrm{~mm} \mathrm{Hg}$ and or a diastolic pressure ranging from 90 to $99 \mathrm{~mm} \mathrm{Hg}$ was classified as stage 1 hypertension, and an average systolic pressure of $160 \mathrm{~mm} \mathrm{Hg}$ or higher and or a diastolic pressure of $100 \mathrm{~mm} \mathrm{Hg}$ or higher as stage 2 .

The two digital photographs of each patient were examined in a blinded fashion by 2 observers (one of the authors and one dentist not involved in the study) not having knowledge about the other's results nor the patient's blood pressure or health status. Both observers viewed the photographs using the same screen. All photographs were graded as none/few visible sublingual varices (grade 0; Fig. 1) or medium/severe sublingual varices (grade 1; Fig. 2). Consensus was reached in cases where the initial assessment differed between observers.

\section{Statistical analysis}

The variables from the questionnaire (sex, actual smoking, hypertension, atrial fibrillation, ischemic heart diseases, myocardial infarction, stroke, other cardiovascular diseases, lower limb varices) were coded as dummy variables. All statistical analyses were performed using IBM SPSS Statistics version 20. Pearson's Chi-square test, Student's t-test, and multiple logistic regression analysis were applied. Correlation between observers was analysed using Cohen's kappa coefficient. Descriptive statistics with index of validity, sensitivity and specificity were also used. All tests were two-sided and the significance cut-off was set at 0.05 .

The expected prevalence of hypertensive patients in a Swedish population above 40 years of age is $38 \%$ [14]. The prevalence of sublingual varices in patients with cardiovascular disease is approximately $70 \%$, compared with $27 \%$ for patients without cardiovascular disease [9]. Power calculations using these data as input resulted in a sample size of 323 patients, considering an alpha error of 0.05 and a beta error of 0.20 . 


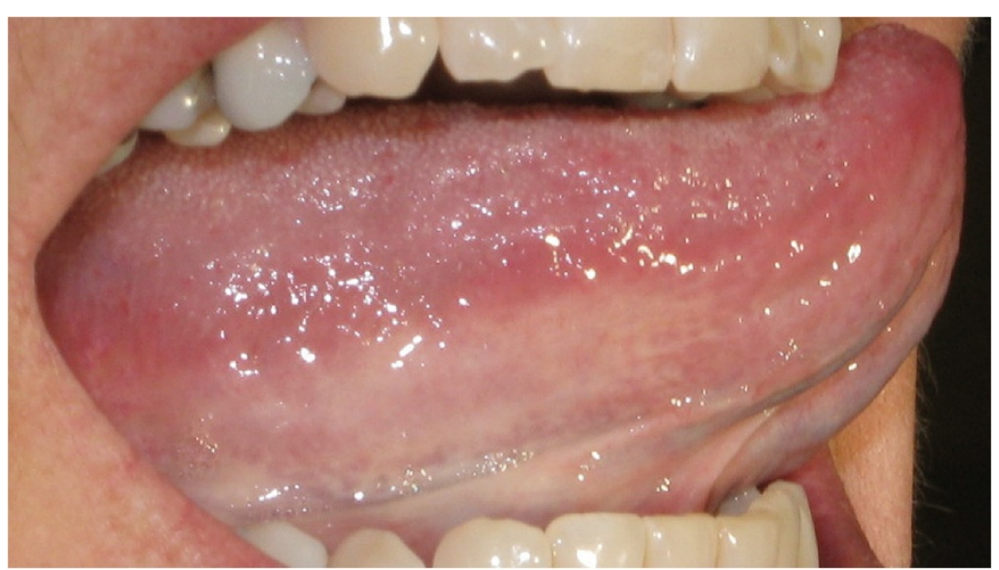

Fig. 1 None/few sublingual varices (grade 0). Written consent obtained from patient

\section{Results}

A total number of 454 patients were invited to participate in the study. Seventeen patients

(3.7\%) resigned from the study (fifteen patients did not want to enter the study, two patients were excluded due to atrial fibrillation). Of the remaining 437 patients, 2 were excluded due to lack of photographs of the tongue, and 4 patients refused to visit the district nurse for follow-up after initial high blood pressure was observed at inclusion. In total 431 patients underwent the complete study protocol. The total dropout number was thus 23 patients (5.1\%; Fig. 3).

The 431 patients who underwent the complete study protocol had a mean age of 55.3 years (SD 10.9). The gender distribution was 188 male and 243 female. The prevalence of smokers was 46 (10.7\%), and 202 (46.9\%) expressed heredity for hypertension. For 92 patients, previously known hypertension was reported (21.3\%) with a mean duration from diagnosis of 7.2 years (SD $7.2)$, and another 28 patients (6.5\%) had other cardiovascular disease (angina pectoris, myocardial infarction or stroke) and 30 patients (7.0\%) had lower limb varices. During the study another 28 participants were identified having hypertension (Fig. 3). A total of 120 (27.8 \%) individuals were thereby hypertensive in the current study.

The prevalence of sublingual varices was $26.5 \%$ (114/ 431) in the study population, with $18.3 \%$ among those having normal blood pressure, and $47.5 \%$ among those diagnosed with hypertension $(p<0.0001)$.

The mean systolic blood pressure measured at inclusion at the dental clinic was $123.2 \mathrm{~mm} \mathrm{Hg}$ (SD 17.3) in the group with grade 0 sublingual varices and $132.1 \mathrm{~mm}$ $\mathrm{Hg}$ (SD 19.3) in the group with grade 1 sublingual varices (Fig. 4; $\mathrm{p}<0.0001$ ). The mean diastolic blood pressure was $79.6 \mathrm{~mm} \mathrm{Hg}$ (SD 12.2) in the group with few or no sublingual varices (grade 0 ) and $83.4 \mathrm{~mm} \mathrm{Hg}$ (SD 13.3) in the grade 1 sublingual varices group (Fig. 5; $p=0.005)$.

The prevalence of grade 1 sublingual varices increased with increasing blood pressure measured at inclusion at the dental clinic, from $21.8 \%$ among those with normal blood pressure to $30.8 \%$ among those with stage 1

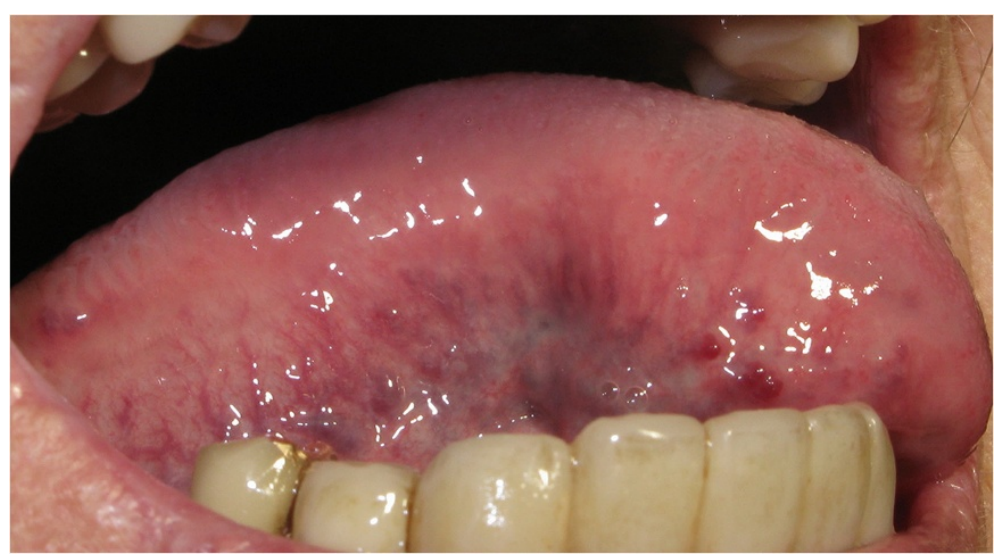

Fig. 2 Moderate/severe sublingual varices (grade 1). Written consent obtained from patient 


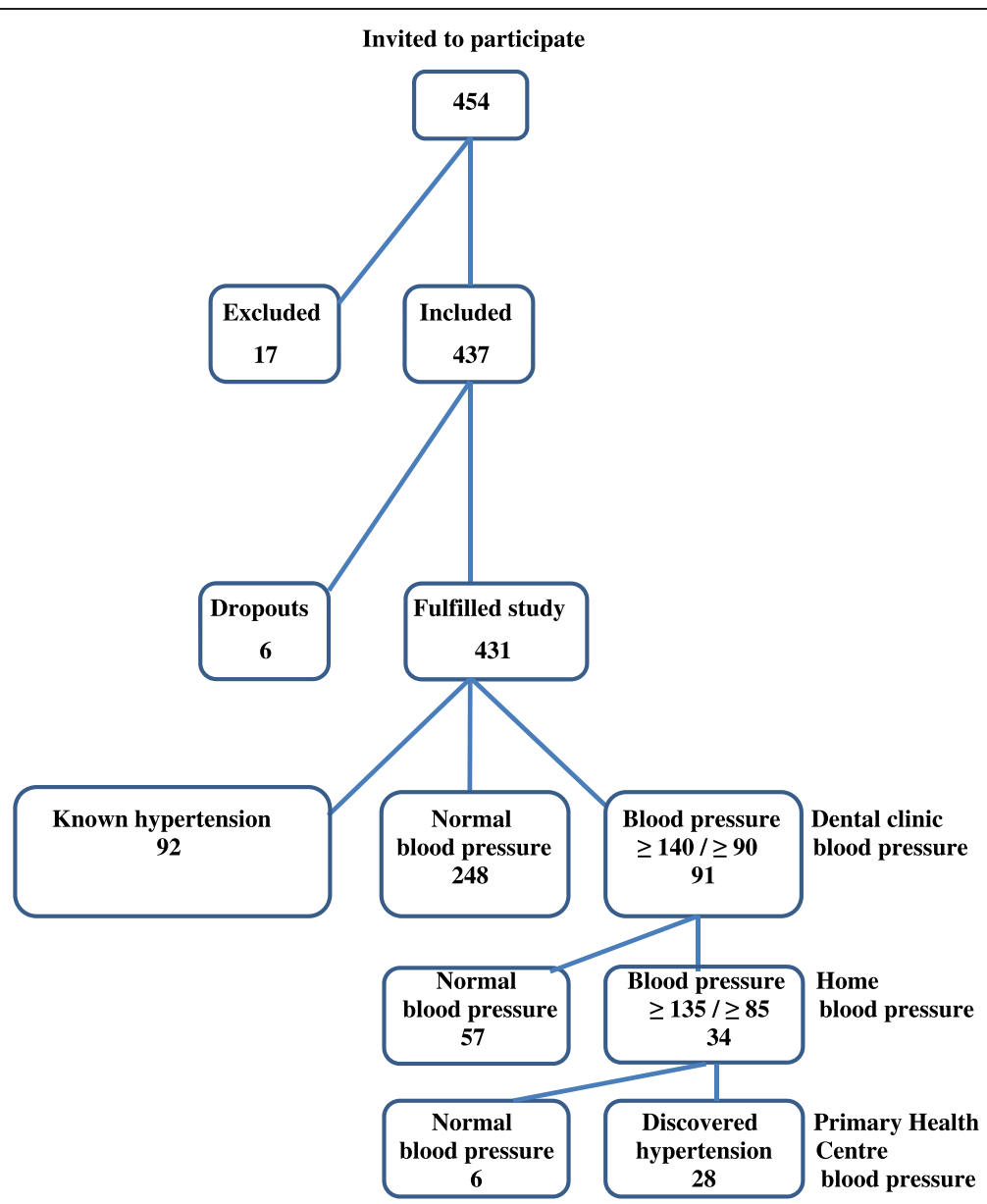

Fig. 3 Flowchart of the study population

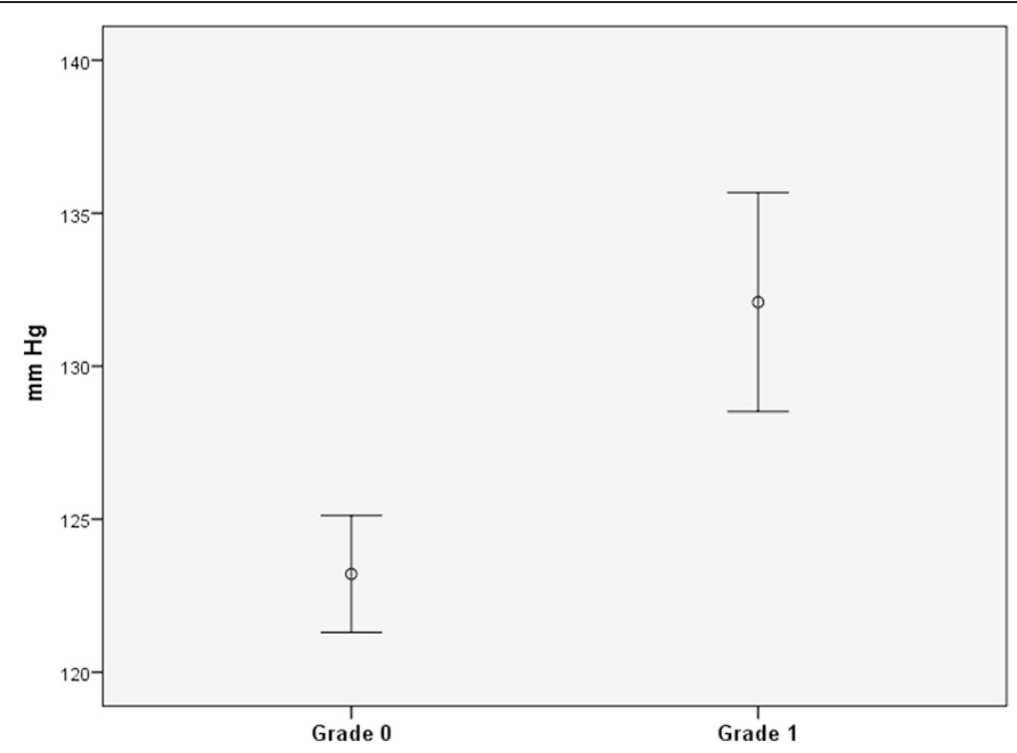

Fig. 4 Mean systolic blood pressure with $95 \%$ confidence intervals in patients with grade $0(n=317)$ and grade $1(n=114)$ sublingual varices, respectively 


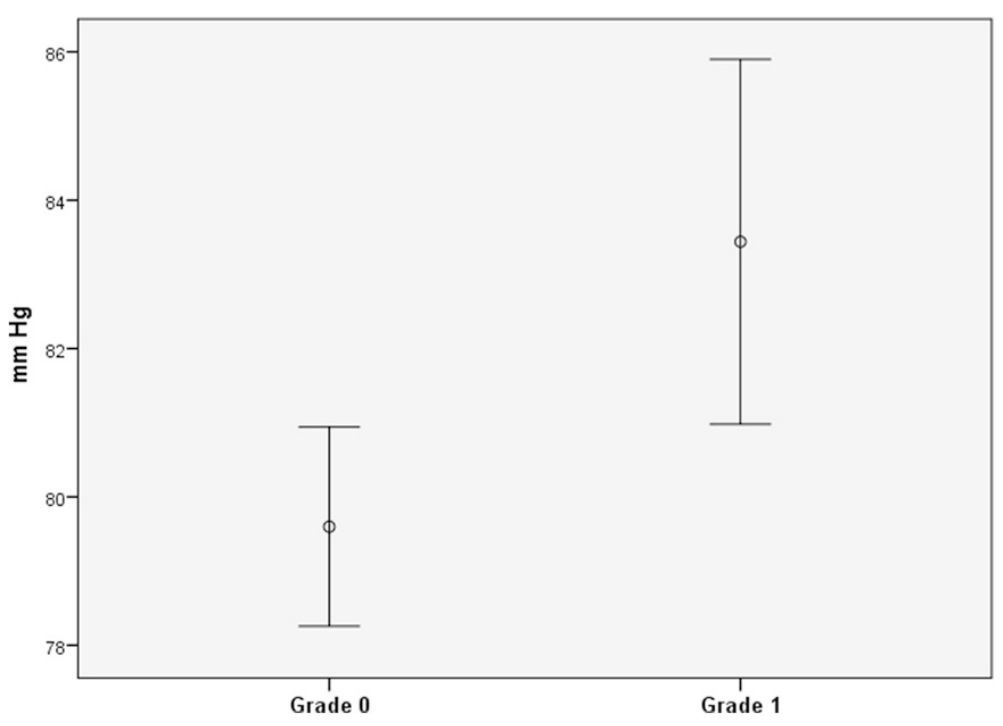

Fig. 5 Mean diastolic blood pressure with $95 \%$ confidence intervals in patients with grade $0(n=317)$ and grade 1 ( $n=114)$ sublingual varices, respectively

hypertension $(p=0.02)$, and further to $43.6 \%$ among those with stage 2 hypertension $(p=0.001)$.

In the group with newly diagnosed hypertension at the primary health care centre $(n=28)$, the prevalence of grade 1 sublingual varices was $46.2 \%$ and $53.3 \%$ in patients with stage 1 hypertension and stage 2 hypertension, respectively.

Age, smoking and hypertension were associated with sublingual varices (Table 1 ). The prevalence of sublingual varices among non-smokers with hypertension was $44.7 \%(46 / 103)$, and among smokers with hypertension $64.7 \%(11 / 17)$.

The identification of sublingual varices as an indicator of hypertension would result in a sensitivity of 0.48 , a specificity of 0.82 , a positive predictive value (PPV) of 0.5 , and a negative predictive value (NPV) of 0.80 (Table 2). Sublingual varices as an indicator of hypertension in the subgroup of smokers showed a sensitivity of 0.65 , a specificity of 0.2 , a PPV of 0.58 , and an NPV of 0.25 .

The inter-observer agreement regarding photographs of the tongue, expressed as index of validity, was 0.87 and the Cohen's kappa coefficient was 0.68.

Table 1 Sublingual varices as the dependent variable (odds ratio $(\mathrm{OR}), \mathrm{p}$-value and $95 \%$ confidence interval $(\mathrm{Cl})$ ) by logistic regression $(n=431)$, adjusted for cardiovascular disease and lower limb varices

\begin{tabular}{llcl}
\hline Variable & OR & $p$-value & $95 \% \mathrm{Cl}$ \\
\hline Age & 1.07 & $<0.0001$ & $1.04-1.09$ \\
Smoking & 2.22 & 0.025 & $1.11-4.45$ \\
Hypertension & 2.25 & 0.002 & $1.34-3.77$ \\
\hline
\end{tabular}

\section{Discussion}

An association between sublingual varices and smoking, age and hypertension was found. The prevalence of sublingual varices increased with stage of hypertension, further confirming the connection to increased blood pressure. The correlation between sublingual varices and hypertension has not been previously shown.

Investigations regarding sublingual varices have earlier been related to histopathology [3, 4], pathogenesis [5], and to the relation between sublingual varices and cardiovascular disease $[6,7]$. In a survey from 1968 Bhaskar et al. [15] could not reveal a correlation between sublingual varices and hypertension, based on 384 cases. In a retrospective study including 281 adults, a strong correlation was found between sublingual varices and cardiovascular disease. The majority of patients with cardiovascular disease in that study had a diagnosis of hypertension (74 \%). The diagnosis of hypertension was however selfreported and not verified by blood pressure measured [9].

In the current study the prevalence of sublingual varices was higher in patients with hypertension diagnosed at the primary health care centre compared to those classified as hypertensive at the dental clinic. This difference may in part be explained by falsely high blood

Table 2 Distribution of sublingual varices in hypertensive and normotensive patients $(n=431)$

\begin{tabular}{lll}
\hline & Hypertensive & Normotensive \\
& $\mathrm{n}(\%)$ & $\mathrm{n}(\%)$ \\
\hline Sublingual varices grade 0 & $63(19.9)$ & $254(80.1)$ \\
Sublingual varices grade 1 & $57(50.0)$ & $57(50.0)$ \\
& 120 & 311 \\
\hline
\end{tabular}


pressure acquired at the dental clinic due to the whitecoat effect, as these patients were found to be normotensive by home blood pressure. Possible explanations to the connection between sublingual varices and hypertension could include circulatory anastomosis in the venous system of the tongue, though there are divergent opinions in the literature [2], or a hemodynamic effect where the arterial pressure influences the veins through arteriovenous shunts [16].

The low drop-out frequency of $5.1 \%$ strengthens the results. A potential weakness of the study lies in estimating whether a patient has sublingual varices. To minimise this potential issue, standardised digital photographs were acquired. The high inter-observer agreement also indicates the effect of this standardisation [17].

A clinical implication of this study is the possibility to use sublingual varices as an indicator of risk for hypertension. Examining the lateral borders of the tongue in at the yearly dental visit is easily and quickly performed, and causes no harm for the patient. Screening for hypertension using identification of sublingual varices (grade 1) in the current study population would identify $48 \%$ of hypertensive patients, with a high specificity of 0.82 .

Based on the results of the current study, a patient above 40 years of age with sublingual varices has a $50 \%$ risk of being hypertensive, and if also being a smoker the risk increases to $58 \%$. If sublingual varices are not present the chance of being normotensive is $80 \%$. The high positive predictive value for hypertension, combined with no discomfort for the patient and minor effort for the dentist, makes sublingual varices highly interesting as a clinical sign to note in the oral status.

\section{Conclusion}

An association was found between sublingual varices and hypertension in patients over 40 years of age. With a sensitivity of 0.48 , a specificity of 0.82 , a PPV of 0.5 , and an NPV of 0.80 the identification of sublingual varices is highly interesting as an indicator of hypertension. Examining the lateral borders of the tongue is easily carried out by a dentist, causes no harm to the patient, and could be a valuable method for the dental profession to take active part in preventive healthcare.

\section{Competing interests}

The authors declare that they have no competing interests.

\section{Acknowledgement}

This study was supported by grants from the Research \& Development Unit, County of Halland, Sweden. Special thanks to Dr. Kerstin Hattne for examining the digital photographs.

We would also like to express our thanks to the manager Britt Lindau and staff at the Public Dental Clinic, Varberg, Sweden.

\section{Author details}

${ }^{1}$ Public Dental Services, Box 1254, S-43218 Varberg, Sweden. ${ }^{2}$ Research \& Development Unit, County of Halland, 30185 Halmstad, Sweden.

Received: 26 October 2014 Accepted: 3 June 2015

Published online: 11 July 2015

\section{References}

1. Lim SS. A comparative risk assessment of burden of disease and injury attributable to 67 risk factors and risk factor clusters in 21 regions, 1990-2010; a analysis for the Global Burden of Disease Study 2010. Lancet. 2012;15:2224-60.

2. Lazos JP, Piemonte ED, Panico RL. Oral varix: a review. Gerodontology. 2013. Epub ahead of print.

3. Rappaport I, Melvin A, Shiffman MD. The significance of oral angiomas. Oral Surg Oral Med Oral Pathol. 1964;17:263-70.

4. Kocsard E, Ofner F, d'Abrera VSE. The histopathology of caviar tongue Ageing changes of the undersurface of the tongue. Dermatologica. 1970;140:318-22.

5. Southham JC, Ettinger RL. A histological study of sublingual varices. Oral Surg Dec. 1974;879-86.

6. Ettinger RL, Mandersson RD. A clinical study of sublingual varices. Oral Surg. 1974;38:540-5

7. Kleinman H. Lingual varicosities. Oral Surg Oral Med Oral Pathol. 1967;23(4):546-8

8. Jassar $\mathrm{P}$, Jaramillo $M$, Nunez DA. Base of tongue varices associated with portal hypertension. Postgrad Med J. 2000;76:576-7.

9. Hedström $\mathrm{L}$, Bergh $\mathrm{H}$. Sublingual varices in relation to smoking and cardiovascular diseases. Br J Oral Maxillofac Surg. 2010;48:136-8.

10. Pickering TG, Miller NH, Ogedegbe G, Krakoff LR, Artinian NT, Goff D. Call to action on use and reimbursement for home blood pressure monitoring: executive summary: a joint scientific statement from the American Heart Association, American Society of Hypertension and Preventive Cardiovascular Nurses Association. Hypertension. 2008;52:1-9.

11. Belghazi J, El Feghali RN, Moussalem T, Rejdych M, Asmar RG. Validation of four automatic devices for self-measurement of blood pressure according to the International Protocol of the European Society of Hypertension. Vasc Health Risk Manag. 2007:3(4):389-400.

12. Parati G, Stergiou GS, Asmar R, Bilo G, de Leeuw P, Imai $Y$, et al. European Society of Hypertension guidelines for blood pressure monitoring at home: a summary report of the Second International Consensus Conference on Home Blood Pressure Monitoring. J Hypertension. 2008;26:1505-26.

13. Mancia G, De Backer G, Dominiczak A, Cifkova R, Fagard R, Germano G, et al. Guidelines for the Management of Arterial Hypertension: The Task Force for the Management or Arterial Hypertension of the European Society of Hypertension (ESH) and of the European Society of cardiology (ESC). J Hypertension. 2007;25:1105-87.

14. SBU-Swedish Council on Health Technology Assessment. Moderately Elevated Blood Pressure- a systematic literature review. Stockholm: SBU; 2004.

15 Bhaskar SN. Oral lesions in the aged population. Geriatrics. 1968:23:137-49.

16 The microcirculation and the lymphatic system: capillary fluid exchange, interstitial fluid, and lymph flow. Local and humoral control of blood flow by the tissues. Guyton AC, Hall JE, editors. Textbook of medical physiology. $11^{\text {th }}$ ed. New York: WB Saunders. 2003. 181-203.

17 Altman DG. Some common problems in medical research. In: Altman DG, editor. Practical statistics for medical research. London: Chapman \& Hall; 1991. p. 396-439

\section{Authors' contributions}

All authors made substantial contributions to conception, design and interpretation of data. MA and LH were involved in acquisition of data. HB generated the figures and tables. All authors were involved in drafting the manuscript and for revising. All authors read and have given final approval of the version to be published. 